\title{
sciendo
}

\section{CLONING AND EXPRESSION LEVELS OF TFAM AND TFB2M GENES AND THEIR CORRELATION WITH MEAT AND CARCASS QUALITY TRAITS IN JIAXING BLACK PIG*}

\author{
Qianqian Song ${ }^{1 *}$, Wei Zhang ${ }^{1}$, Fen $\mathrm{Wu}^{1 *}$, Jinzhi Zhang ${ }^{1 *}$, Mingshu Xu ${ }^{2}$, Haihong $\mathrm{Li}^{2}$, Zhujun $\mathrm{Han}^{2}$, \\ Haixia $\mathrm{Gao}^{2}$, Shanlin $\mathrm{Zhao}^{2}$

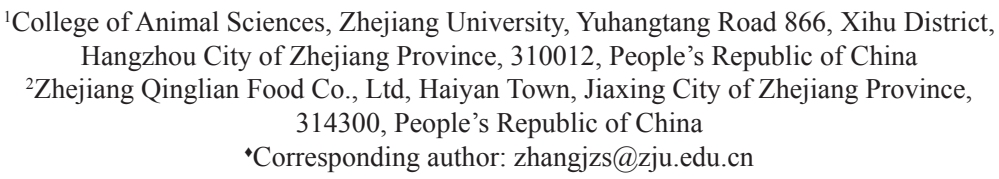

\begin{abstract}
The coding sequences (CDS) of $T F A M$ and $T F B 2 M$ genes from Jiaxing Black Pig (JBP) were first obtained by RT-PCR and DNA-seq in the present study. Sequence analyses showed that the TFAM gene contains a 741-bp CDS region encoding 246 amino acids sharing a $100 \%$ homology with the sequence on NCBI, while $T F B 2 M$ gene contains a CDS region of 1176 bp encoding 391 amino acids with two missense mutations. The results of quantitative Real-Time PCR for TFAM and TFB2M revealed that transcripts of the genes were both presented at the highest levels in spleen tissue followed by liver tissue, while the least levels in longissimus dorsi muscle (LDM), and obviously the higher levels in two adipose tissues than those in LDM tissue $(P<0.01)$. Meanwhile, a total of forty-two JBPs were employed in this experiment to investigate the effect of these two genes on the carcass, meat quality traits and flavor substances such as fatty acids, intramuscular fat (IMF) in LDM. As expected, some strong correlations of gene expression abundance of $T F A M$ and $T F B 2 M$ mRNA in particular tissues such as liver and LDM with carcass and meat quality traits including marbling score, as well as the content of saturated fatty acid (SFA), in JBP were found.
\end{abstract}

Key words: cloning, Jiaxing Black Pig, TFAM and $T F B 2 M$, mRNA expression, carcass and meat quality traits

Jiaxing Black Pig (JBP), one native member of Taihu pig currently classified into six sub-breeds (namely, Meishan, Jiaxing Black, Fengjing, Shawutou, Erhualian, and Mizhu) according to the most recently reported classification of Chinese indigenous swine breeds (China National Commission of Animal Genetic Resources, 2011), has been renowned for its high prolificacy and excellent meat quality characteristics (Wang et al., 2017).

\footnotetext{
*These authors contributed equally to this work.
} 
TFAM (mitochondrial transcription factor A, mtTFA) and TFB2M (mitochondrial transcription factor B2) compose the mitochondrial transcription basic machine along with TFB1M (mitochondrial transcription factor B1) and POLRMT (mitochondrial RNA polymerase) (Falkenberg et al., 2002; Fisher and Clayton, 1988). TFAM binding to mitochondrial DNA (mtDNA), a high mobility group class protein encoded by the nuclear genome, protects a region 14-35 bp upstream of the light strand promoter transcription start site, and assists in assembly of the initiation complex by attracting POLRMT and other transcription factors (Dairaghi et al., 1995). TFB2M regulating mitochondrial transcription, a bifunctional protein encoded by the nuclear genome, is one of the rRNA methyltransferases, whose expression is strictly regulated by nuclear transcription factors, such as NRF-1, NRF-2 and PGC-1 $\alpha$ (Yakubovskaya et al., 2010; Rebelo et al., 2011). However, some studies demonstrated that only TFAM, $T F B 2 M$ and POLRMT are required for initiation of transcription of mtDNA since $T F B 2 M$ as a transcription factor has much more activity than TFB1M (Litonin et al., 2010; Rantanen et al., 2003).

In recent years, the number of investigations on the effect of TFAM and TFB2M genes on animals' production have been increasing due to the biological functions of the said genes in relation to a number of traits which have proven to be of economic significance. In the studies on cattle, two SNPs situated in the promoter region of TFAM gene were revealed to have significant impacts on marbling and subcutaneous fat depth and to cause changes of transcription factor binding sites associated with fat deposition and energy metabolism (Jiang et al., 2005) and three nuclear-encoded mitochondrial genes such as TFAM, FABP4 and mitochondrial polymerase A were found to be significantly associated with marbling (Jiang et al., 2009). Fernandez et al. (2008) demonstrated that the polymorphism of mtDNA had an effect on the meat quality traits such as IMF and protein content in Iberian pigs. Cui et al. (2015) studied the expression of TFB2M gene in Guizhou white goat tissues, speculating that the gene may play a role in fat deposition based on previous studies.

However, thus far, little is known about TFAM and TFB2M genes related to meat quality traits in JBP. Our investigation aimed to find potential favorable molecular markers or candidate genes involved with pork quality, which could provide a future theoretical basis for improving pork quality and speeding up the JBP breeding process.

\section{Material and methods}

\section{Experimental animals and tissue collection}

The research was undertaken in strict accordance with institutional guidelines proposed by the China Council on Animal Care. All animal procedures were conducted with the approval of the Laboratory Animal Center of Zhejiang University (Hangzhou, China). Data was obtained from the forty-two healthy pigs of similar weight $(71 \pm 2 \mathrm{~kg})$ and age ( $210 \pm 5$ days) provided by Zhejiang Qinglian Food Co., Ltd. Upon reaching commercial slaughter weight the selected animals were then fasted, leaving them with access only to water. The live weight (LW) was determined 
$24 \mathrm{~h}$ prior to slaughtering at a commercial abattoir. Fresh samples of heart, liver, spleen, lung, stomach, LDM, abdominal (AF) and subcutaneous (SF) fat tissues of JBP were harvested immediately after slaughter. The samples were stored in liquid nitrogen and then stored at $-80^{\circ} \mathrm{C}$ for RNA extraction.

\section{Traits measurement}

Recordings made for each carcass included the chilled carcass weight (CCW) after being kept at a temperature of $4^{\circ} \mathrm{C}$ for $24 \mathrm{~h}$. The weight of various carcass components (lean, bone and subcutaneous fat including skin) were recorded. The dressing out percentage (DOP), bone, lean and fat ratios were calculated, respectively. The average back fat thickness (BFT) was determined in the midline on the scapula back edge, last rib and last lumbar vertebrae with a sliding caliper, while the skin thickness (ST) at the 6 7 rib of the centerline of the carcass, and simultaneously the fat thickness $\left(\mathrm{FT}_{6 \sim 7}\right)$ was recorded at this point. Ham-to-Carcass Ratio (HCR) was assessed by recording left ham weight and calculating the percentage of ham. The loin eye muscle area (LEMA) was evaluated at the last rib of vertical LDM cut by tracing its surface area covered with cross section of sulfuric acid paper.

The LDM adjacent to the last rib was taken for evaluating meat quality traits. The $\mathrm{pH}$ at 45 min post mortem in LDM was also measured by $\mathrm{pH}-\mathrm{Star}$ (Matthäus, Germany) at the level of the 6th rib and recorded. The water holding capacity (WHC) was estimated according to filter paper fluid uptake as described by Kauffman et al. (1986). Meat color and marbling scores were subjectively evaluated based on color standard $(1=$ pale; $6=$ dark $)$ and marbling grade standards provided by US National Pork Procedures Council (NPPC). A reflectance spectrophotometer (X-Rite SP64, USA) was used to measure the lightness $\left(L^{*}\right)$ and two color coordinates (redness, $a^{*}$; yellowness, $b^{*}$ ). Tenderness of the meat was measured through Warner-Bratzler shear force value expressed as $\mathrm{kg}$ of force (kgf).

\section{Measurements of flavor substances}

Content of inosinic acids (IMP) extracted with $0.5 \mathrm{M}$ perchloric acid from meat ground in liquid nitrogen was detected using high-performance liquid chromatography (HPLC) and calculated according to peak areas of sample fluid and the standard liquid. IMF content of LDM tissue was extracted and examined according to the procedures of AOAC (1990). Fatty acid composition was analyzed by gas chromatography (Agilent 6820, Agilent Technologies, USA) according to the method described by Yang et al. (2005). Amino acid composition of the muscle powder was analyzed using ion-exchange chromatography with an automatic amino acid analyzer (S-433D Sykam Automatic Amino Acid Analyzer, Germany).

\section{Total RNA extraction and cDNA preparation}

Total RNA was isolated from JBP tissue samples with TRIZOL reagent (Invitrogen Corporation, Carlsbad, California) according to the instructions of the manufacturer. For each sample, $1 \mu \mathrm{g}$ of qualified total RNA was used to be reversely transcribed into the first cDNA using Prime Script ${ }^{\text {TM }}$ RT reagent Kit with gDNA Eraser (Takara Biotechnology Co. Ltd., Dalian, China). 


\section{Cloning and sequence analyses of CDS of TFAM and TFB2M genes}

Two sets of gene-specific primers of the genes were designed with Primer 5.0 according to the TFAM gene sequences (GenBank accession No. NM_001130211.1) and TFB2M gene sequences (GenBank accession XM_021064472.1). The forward and the reverse primers of two genes (TFAM-F1: TTGCGAGTTCAAGTCGTCAT, TFAM-R1: GGTTTCTCGTGTCTATCCAT; TFB2M-F1: GTGGGTCGTCCTGTATGG, TFB2M-R1: ACACATGGCACAGGGAGA) were synthesized by TSINGKE Biotech Co. Ltd. (Beijing, China). The expected amplicon lengths of TFAM and TFB2M were 943 bp and 1723 bp, respectively. The PCR products were identified with $1 \%$ agarose gel electrophoresis and then the purified PCR products using DNA Gel extraction kit (Takara, China) were cloned into the pMD19-T Simple Vector and sequenced (TSINGKE, China).

The sequencing results of the genes were analyzed by Seqman in DNASTAR (DNASTAR, Inc., Wisconsin, USA) software. The open reading frames (ORF) were found using the ORF Finder (www.ncbi.nlm.nih.gov/gorf/). The analysis of the conserved domain was conducted by SMART search program (http://smart.emblheidelberg.de/).

\section{Quantitative Real-Time PCR (qRT-PCR)}

The expression levels of TFAM and TFB2M of nine different tissue samples taken from the selected (JBP) animals were determined by qRT-PCR assay. Primers of the target genes were designed according to the TFAM and TFB2M mRNA sequence cloned. The glyceraldehyde phosphate dehydrogenase $(G A P D H)$, a housekeeping gene, was selected as an internal control and amplified with primers designed based on the gene sequence (GenBank NM_001206359.1) (Table 1). The SYBR Green qRT- PCR assay was performed on a qRT- PCR machine (ABI700 Real-Time System, USA). Melting curve analyses were performed at the end of PCR to check the specificity of the PCR reaction. For each tissue, qRT-PCR amplification was performed in triplicate.

Table 1. Real time PCR primer information

\begin{tabular}{llcc}
\hline \multicolumn{1}{c}{ Primer } & \multicolumn{1}{c}{ Primer sequence $\left(5^{\prime} \rightarrow 3^{\prime}\right)$} & $\begin{array}{c}\text { Annealing temperature } \\
\left({ }^{\circ} \mathrm{C}\right)\end{array}$ & $\begin{array}{c}\text { Amplified DNA } \\
\text { fragment }(\mathrm{bp})\end{array}$ \\
\hline TFAM-RT-F & CTCTCCGTTCAGTTTTGCGT & 60 & 136 \\
TFAM-RT-R & TGCATCTGGGTTCTGAGCTT & & 175 \\
TFB2M-RT-F & TGGATTCCTGACACGAGCAT & 60 & 100 \\
TFB2M-RT-R & AGGTGTTAGGGCTCCATGAC & & \\
GAPDH-RT-F & ACTCACTCTTCTACCTTTGATGCT & 60 & \\
GAPDH-RT-R & TGTTGCTGTAGCCAAATTCA & & \\
\hline
\end{tabular}

\section{Statistical analysis}

Final relative quantification of target gene expression was calculated utilizing the $2^{-\triangle \Delta C T}$ method (Livak and Schmittgen, 2001). All experimental data are presented as the mean \pm standard error, analyzed by One-Way ANOVA procedure of SPSS 20.0. 
Comparisons of the mRNA expression values of the genes among tissues were made by the least significant difference (LSD) and the Duncan test as appropriate. Differences were considered significant when $\mathrm{P}<0.05$. Finally, the correlation coefficients of TFAM and TFB2M mRNA expression with the carcass and meat quality traits of JBP were also analyzed by bivariate correlation of SPSS software, respectively.

\section{Results}

\section{Cloning and sequence analyses of the CDS of TFAM and TFB2M}

The cloned PCR products of TFAM and TFB2M are 943bp and 1723bp in length with CDS regions of $741 \mathrm{bp}$ and $1176 \mathrm{bp}$ encoding 246 amino acids and 391 amino acids, respectively. Sequencing analyses found that CDS of TFB2M gene of JBP shares a $99.57 \%$ homology with the nucleotide sequence of Sus scrofa CDS region submitted on NCBI. There were five mutations detected in CDS of TFB2M in JBP (Accession number:MK105895), including G294A, G552C, G984A, G985A and G1012A, but with only two amino acids changing both from valine to isoleucine at 329th and 338th site (Figure 1 and 2).

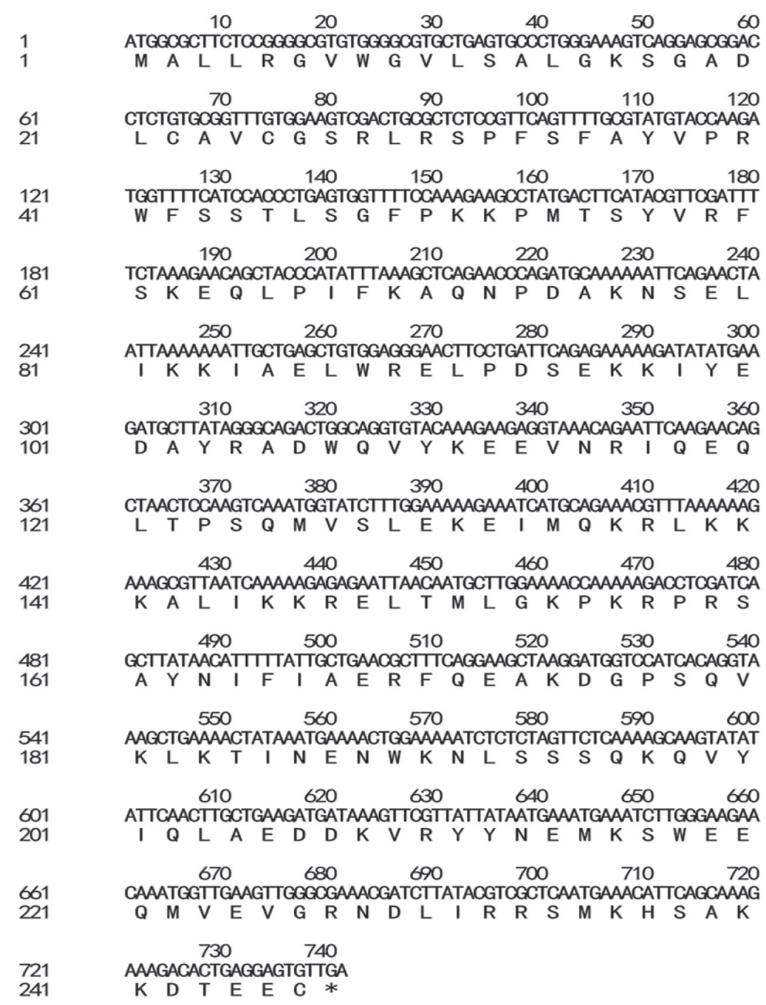

Figure 1. The nucleotide and amino acid sequence of TFAM in JBP 


\begin{tabular}{|c|c|}
\hline & 60 \\
\hline 1 & АTGTGGGTOOCATGGGOGQGGCTTOCAOCAOGGCTAAOGCTCTCACOCTTGAOOGTOOCTGGQOCCTTाTGCACT \\
\hline & 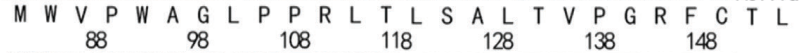 \\
\hline & AGGTOOGGAGOGCCAAGGAGGAAGGATGTTOOGGOGGGGCAOOCOOGTGOCTTGTCTGATTTOCAACOGAAGCTG \\
\hline & 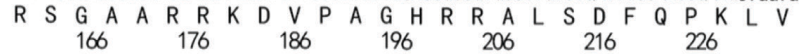 \\
\hline & ООСTGTGTGGGTTIOGGGAAGTOGOGTGTGTACAAGCACAACTCAGAAOOCAAGOGGTACATAACTAATOOGAGAC \\
\hline & 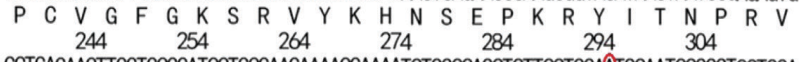 \\
\hline & GCTGAGAACTTGGTGOGGATCCTGOGAAGAAAACGAAAATCTGGOCAGCTCTTOCTGGAÄTGCAATCOGGGTOCTC \\
\hline & 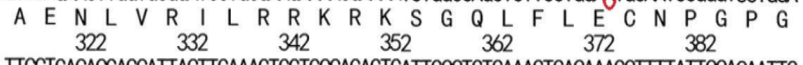 \\
\hline & TTOCTGACACGAGCATTACTTGAAAGTGGTGCCAGAGTGATTGOCTGTGAAAGTGACAAAGCTाTATTCCACAATTG \\
\hline & 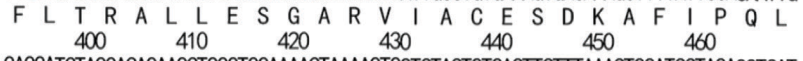 \\
\hline & GAGGATCTAGGACAGAAGCTGGGTGGAAAACTAAAAGTGGTCTACTGTGACTTCTITAAACTGGATOCTAGAGGT \\
\hline & 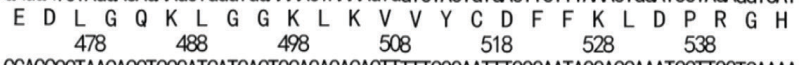 \\
\hline & GGAGOOCTAACAOCTCOGATCATGACTGCAGAGACACTITTOGGAATTTGGGAATAGGAOCAAATCCTTGGTCAAAA \\
\hline & 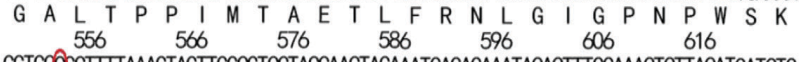 \\
\hline & GGTGQGGCTITAAAGTAGTTGGOGTCCTACCAACTAGAAATGAGAGAAATACACTITGGAACTCTTACATGATCTG \\
\hline & 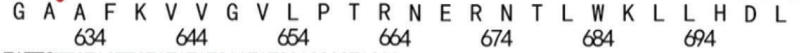 \\
\hline & TATTCTTGTACTTCTATATATGAATATGGAOGGGTAGAAGTAAACATGTTTGTTACOGAAAAAGAATGCOGGAAAATA \\
\hline & 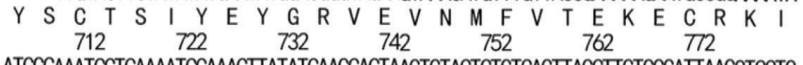 \\
\hline & ATGGCAAATOCTCAAAATOCAAACTTATATCAAGCACTAAGTGTACTCTGTCAGTTAGCTTGTGGGATTAAGGTOCTG \\
\hline & 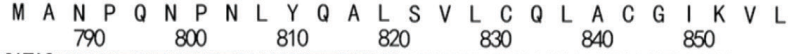 \\
\hline & CATACGGAGOCTTGCTCATCATTCAGAACATTTATOCAAAATGGGCAGCTGGAAAAGAAGCAGCATAGGGAATCAC \\
\hline & 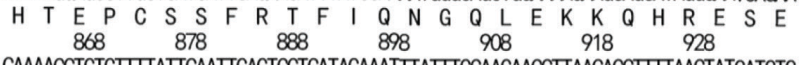 \\
\hline & 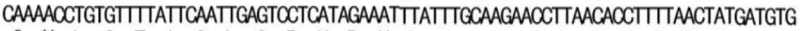 \\
\hline 7 & 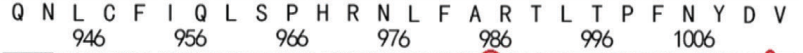 \\
\hline & ПIIITCACATGGTAAGGCAGTGTITATGAAACGCAAOGCCATGCTAATAGACCATTTACGTTCATTGAGTOCAAПT \\
\hline & 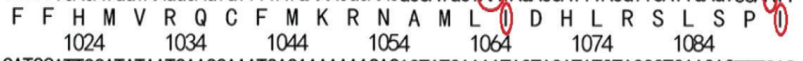 \\
\hline & GATGCATTGCATATAATGAAOCAAATGAGAAAAAAACACAGTATGAAAATAGTAGATATGTACOCTGAAGACTITCAG \\
\hline & 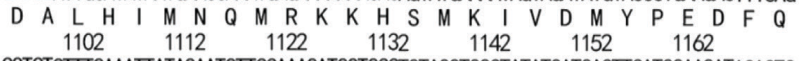 \\
\hline & OGTCTCTITGAAATTATAGAATGTTOCAAAGATGGTGOCTGTAGGTGCCTATATGATGACTTCATGGAAGATACACTC \\
\hline 65 & 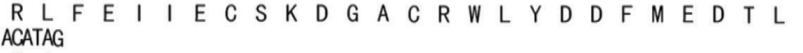 \\
\hline & \\
\hline
\end{tabular}

Figure 2. The nucleotide and amino acid sequence of TFB2M from JBP. Differences of the sequences were marked with red circles in the figure

\section{Predicted amino acid sequence analysis of $T F A M$ and $T F B 2 M$}

The TFAM protein of JBP contains twenty kinds of amino acids, of which the highest proportion of Lysine is $12.2 \%$, and the lowest proportion of Histidine is $0.4 \%$. For $T F B 2 M$ protein also consists of twenty kinds of amino acids, in which Leucine accounts for the highest content of $11.3 \%$ and Tryptophan for the lowest content of $1.3 \%$. It was also found that there are two HMGs (high mobility group protein) at the locations between 49-119 and 154-220 amino acid residues, in TFAM protein, and a signal peptide at front of the first domain (Figure 3 a). However, the only one domain of ribosomal RNA adenine dimethylases $(r A D c)$ was found in $T F$ $B 2 M$ protein (Figure $3 \mathrm{~b}$ ). 
a

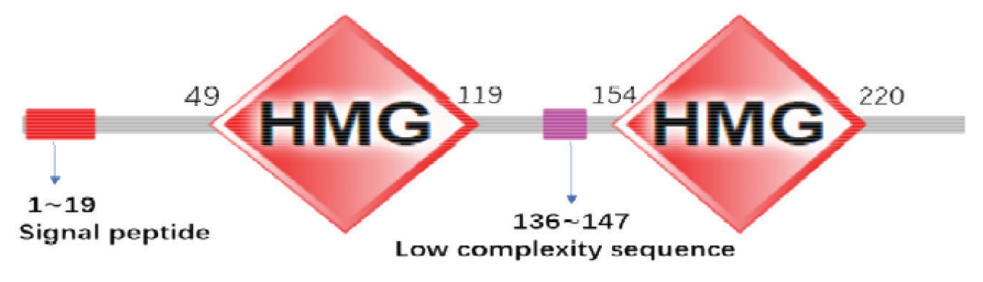

$\begin{array}{lll}0 & 100 & 200\end{array}$

b

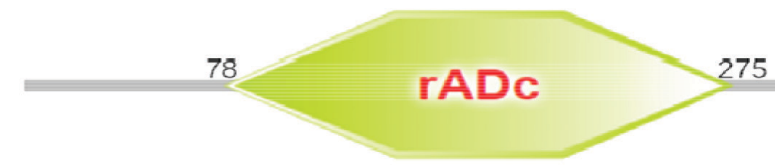

o

100

200

300

Figure 3. Analyses of conservative structure domain of TFAM (a) and TFB2M (b)

a

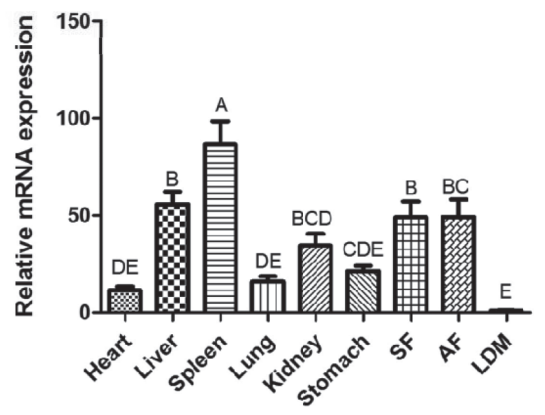

b

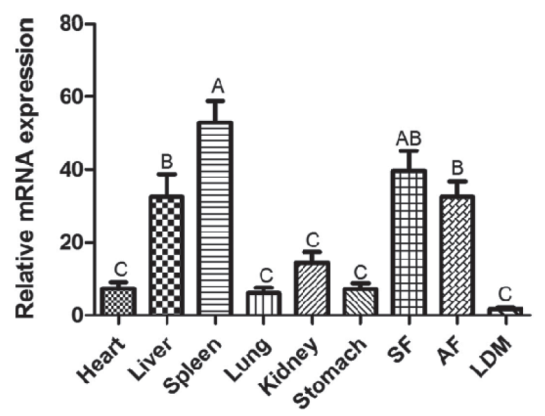

Columns with different superscript capital letters differ highly significantly $(\mathrm{P}<0.01)$.

Figure 4. Expression of TFAM gene (a) and TFB2M gene (b) in different tissues of JBP 
Table 2. The descriptive statistics of the carcass and meat quality traits in JBP pigs

\begin{tabular}{|c|c|}
\hline Traits & Mean \pm SE \\
\hline LW (kg) & $72.26 \pm 0.75$ \\
\hline $\mathrm{CCW}(\mathrm{kg})$ & $50.16 \pm 0.62$ \\
\hline $\mathrm{BFT}(\mathrm{cm})$ & $2.92 \pm 0.05$ \\
\hline $\mathrm{FT}_{6 \sim 7}(\mathrm{~cm})$ & $2.94 \pm 0.06$ \\
\hline $\mathrm{ST}(\mathrm{cm})$ & $0.50 \pm 0.01$ \\
\hline LEMA $\left(\mathrm{cm}^{2}\right)$ & $16.65 \pm 0.22$ \\
\hline HCR (\%) & $28.20 \pm 0.23$ \\
\hline DOP (\%) & $69.38 \pm 0.31$ \\
\hline LP (\%) & $39.45 \pm 0.24$ \\
\hline BP $(\%)$ & $10.34 \pm 0.12$ \\
\hline FP $(\%)$ & $50.21 \pm 0.28$ \\
\hline $\mathrm{pH}$ & $6.36 \pm 0.02$ \\
\hline Meat color score & $3.28 \pm 0.04$ \\
\hline Marbling score & $3.36 \pm 0.03$ \\
\hline Water loss rate & $12.57 \pm 0.16$ \\
\hline Shear force & $16.47 \pm 0.28$ \\
\hline$L^{*}$ & $48.09 \pm 0.29$ \\
\hline$a^{*}$ & $2.91 \pm 0.13$ \\
\hline$b^{*}$ & $12.39 \pm 0.22$ \\
\hline IMP & $2.93 \pm 0.05$ \\
\hline IMF & $4.62 \pm 0.14$ \\
\hline Glu & $3.10 \pm 0.02$ \\
\hline TAA & $20.49 \pm 0.08$ \\
\hline $\mathrm{C}_{16: 0}$ & $27.00 \pm 0.04$ \\
\hline $\mathrm{C}_{18: 1}$ & $45.92 \pm 0.06$ \\
\hline SFA & $41.54 \pm 0.03$ \\
\hline TUFA & $58.47 \pm 0.03$ \\
\hline
\end{tabular}

\section{TFAM and TFB2M mRNA expression}

Quantitative real-time PCR normalized against GAPDH levels analyses showed the abundance of TFAM and TFB2M transcript of nine tissues in JBP (Figure 4). The analysis revealed that TFAM and TFB2M were both expressed in all detected tissues with the highest mRNA levels observed in spleen followed by liver, and the lowest level in LDM. And mRNA expression intensity of the two genes in different tissues tended to be spleen $>$ liver $>\mathrm{SF}, \mathrm{AF}>$ kidney $>$ stomach, lung, heart $>$ LDM in general. All the data was only compared at the level of high significance considering the variety of these tissues. For instance, when compared to the levels of TFAM expression in heart, lung and LDM, the ones in liver, spleen, AF and SF were significantly higher $(\mathrm{P}<0.01)$.

\section{The correlations of $T F A M \& T F B 2 M$ expression profiles with carcass traits}

The correlations of TFAM and TFB2M expression levels of JBP with their carcass traits (Table 2) were analyzed by SPSS as shown in Table 3 and Table 4 . There 
were significantly negative correlations between TFAM mRNA expression in liver and BFT along with $\mathrm{FT}_{6 \sim 7}(\mathrm{P}<0.01)$. The expression levels of the TFAM gene in AF as well as SF were highly negatively correlated with LW, CCW, LP and LEMA, whereas strongly positively with FP $(\mathrm{P}<0.05)$, respectively. Remarkably negative correlations between TFAM mRNA expression levels, ST and FT $_{6 \sim 7}$ were observed in LDM. With respect to TFB2M, the mRNA expression in AF was significantly negative in relation to BFT $(\mathrm{P}<0.05)$ as well as $\mathrm{FT}_{6 \sim 7}(\mathrm{P}<0.05)$ that also was found to be obviously linked with mRNA expression in SF $(\mathrm{P}<0.05)$. Lastly, there was a distinctly negative correlation found between ST and TFB2M mRNA expression in $\operatorname{LDM}(\mathrm{P}<0.05)$.

Table 3. The correlation analyses with the expression of TFAM gene and carcass traits

\begin{tabular}{l|c|c|c|c|c|c|c|c|c|c|c}
\hline \multicolumn{10}{c}{ Carcass traits } \\
\hline $\begin{array}{c}\text { Tis- } \\
\text { sues }\end{array}$ & $\begin{array}{c}\text { LW } \\
(\mathrm{kg})\end{array}$ & $\begin{array}{c}\mathrm{CCW} \\
(\mathrm{kg})\end{array}$ & $\begin{array}{c}\text { BFT } \\
(\mathrm{cm})\end{array}$ & $\begin{array}{c}\mathrm{FT}_{6-7} \\
(\mathrm{~cm})\end{array}$ & $\begin{array}{c}\text { ST } \\
(\mathrm{cm})\end{array}$ & $\begin{array}{c}\text { LEMA } \\
\left(\mathrm{cm}^{2}\right)\end{array}$ & $\begin{array}{c}\text { HCR } \\
(\%)\end{array}$ & $\begin{array}{c}\text { DOP } \\
(\%)\end{array}$ & $\begin{array}{c}\text { LP } \\
(\%)\end{array}$ & $\begin{array}{c}\text { BP } \\
(\%)\end{array}$ & $\begin{array}{c}\text { FP } \\
(\%)\end{array}$ \\
\hline Liver & 0.046 & -0.011 & $-0.417 * *$ & $-0.402 * *$ & 0.020 & 0.042 & 0.110 & -0.124 & -0.085 & -0.050 & 0.094 \\
SF & $-0.427 * *-0.404 * *$ & -0.266 & -0.288 & -0.029 & $-0.387 *-0.185$ & -0.092 & $-0.376^{*}$ & -0.119 & $0.374 *$ \\
AF & $-0.456^{* *}-0.377^{*}$ & -0.158 & -0.243 & 0.055 & $-0.373 *-0.243$ & 0.055 & $-0.488^{* *}-0.139$ & $0.478^{* *}$ \\
LDM-0.111 & -0.037 & -0.262 & -0.360 & $-0.313^{*}-0.134$ & -0.226 & $0.166-0.144$ & -0.140 & 0.184 \\
\hline
\end{tabular}

Superscript indicates significant difference $(* \mathrm{P}<0.05 ; * * \mathrm{P}<0.01)$.

$\mathrm{SF}=$ subcutaneous fat; $\mathrm{AF}=$ abdominal fat; $\mathrm{LDM}=$ longissimus dorsi muscle; $\mathrm{LW}=$ live weight; $\mathrm{CCW}=$ chilled carcass weight; BFT $=$ back fat thickness; $\mathrm{ST}=$ skin thickness; $\mathrm{FT}_{6 \sim 7}=$ fat thickness of $6 \sim 7$ th rib of the centerline of the carcass; LEMA = loin eye muscle area; $\mathrm{LP}=$ lean percentage; $\mathrm{BP}=$ bone percentage; $\mathrm{FP}=$ fat percentage.

\section{The correlations of $T F A M$ \& $T F B 2 M$ expression profiles with pork meat quality traits}

The results exhibited in Table 5 revealed that the expression of the TFAM in AF was positively correlated with WHC with reaching the statistical significance $(\mathrm{P}<0.05)$. However, the TFAM and TFB2M expression level in LDM were both intensely correlated with marbling score in a negative way $(\mathrm{P}<0.05)$. Interestingly, $T F B 2 M$ expression in LDM was found to be strongly corresponded with shear force value, $L^{*}$ value $(\mathrm{P}<0.05)$ and $b^{*}$ value $(\mathrm{P}<0.01)$ in Table 6 .

Table 4. The correlation analyses with the expression of TFB2M gene and carcass traits

\begin{tabular}{l|c|c|c|c|c|c|c|c|c|c|c}
\hline \multicolumn{10}{c}{ Carcass traits } \\
\hline Tissues & $\begin{array}{c}\text { LW } \\
(\mathrm{kg})\end{array}$ & $\begin{array}{c}\mathrm{CCW} \\
(\mathrm{kg})\end{array}$ & $\begin{array}{c}\text { BFT } \\
(\mathrm{cm})\end{array}$ & $\begin{array}{c}\mathrm{FT}_{6 \sim 7} \\
(\mathrm{~cm})\end{array}$ & $\begin{array}{c}\text { ST } \\
(\mathrm{cm})\end{array}$ & $\begin{array}{c}\text { LEMA } \\
\left(\mathrm{cm}^{2}\right)\end{array}$ & $\begin{array}{c}\text { HCR } \\
(\%)\end{array}$ & $\begin{array}{c}\text { DOP } \\
(\%)\end{array}$ & $\begin{array}{c}\text { LP } \\
(\%)\end{array}$ & $\begin{array}{c}\text { BP } \\
(\%)\end{array}$ & $\begin{array}{c}\text { FP } \\
(\%)\end{array}$ \\
\hline Liver & 0.168 & 0.027 & -0.269 & -0.255 & -0.024 & 0.165 & 0.236 & -0.301 & 0.019 & 0.075 & -0.048 \\
SF & -0.240 & -0.218 & -0.259 & $-0.331^{*}$ & -0.03 & 0.230 & -0.023 & -0.109 & -0.212 & -0.254 & 0.291 \\
AF & 0.058 & 0.024 & $-0.340^{*}$ & $-0.389^{*}$ & -0.019 & 0.024 & 0.213 & -0.056 & -0.276 & -0.084 & 0.273 \\
LDM & 0.002 & 0.107 & -0.050 & -0.189 & $-0.363^{*}$ & -0.173 & -0.179 & 0.276 & -0.082 & -0.015 & 0.077 \\
\hline
\end{tabular}

Superscript indicates significant difference $(* \mathrm{P}<0.05 ; * * \mathrm{P}<0.01)$. 
Table 5. The correlation analyses with the expression of TFAM gene and meat quality traits

\begin{tabular}{l|c|c|c|c|c|c|c|c}
\hline \multirow{2}{*}{ Tissues } & \multicolumn{7}{c}{ Meat quality traits } \\
\cline { 2 - 9 } & $\mathrm{pH}$ & MCS & MS & WHC & shear force & $L^{*}$ & $a^{*}$ & $b^{*}$ \\
\hline Liver & 0.023 & 0.14 & 0.017 & 0.112 & 0.204 & 0.044 & -0.026 & -0.231 \\
SF & -0.004 & 0.026 & 0.207 & 0.241 & 0.11 & 0.154 & -0.075 & -0.092 \\
AF & 0.067 & 0.165 & 0.129 & $0.327 *$ & 0.079 & 0.107 & -0.001 & -0.212 \\
LDM & 0.028 & 0.143 & $0.392 *$ & 0.164 & 0.145 & 0.01 & -0.267 & 0.106 \\
\hline
\end{tabular}

Superscript indicates significant difference $(* \mathrm{P}<0.05)$.

$\mathrm{MCS}=$ meat color score; $\mathrm{MS}=$ marbling score.

Table 6 . The correlation analyses with the expression of TFB2M gene and meat quality traits

\begin{tabular}{l|c|c|c|c|c|c|c|c}
\hline \multirow{2}{*}{ Tissues } & \multicolumn{8}{|c}{ Meat quality traits } \\
\cline { 2 - 10 } & $\mathrm{pH}$ & MCS & MS & WHC & shear force & $L^{*}$ & $\mathrm{a}^{*}$ & $\mathrm{~b}^{*}$ \\
\hline Liver & 0.204 & 0.049 & 0.001 & -0.146 & 0.074 & -0.133 & 0.006 & 0.281 \\
SF & 0.182 & -0.063 & 0.015 & 0.138 & 0.189 & 0.001 & 0.066 & -0.280 \\
AF & 0.082 & -0.034 & 0.193 & 0.110 & 0.050 & -0.072 & -0.084 & -0.216 \\
LDM & 0.049 & 0.007 & $0.428^{* *}$ & 0.108 & $0.348^{*}$ & $0.342^{*}$ & -0.179 & $0.480^{* *}$ \\
\hline
\end{tabular}

Superscript indicates significant difference $(* \mathrm{P}<0.05)$.

\section{The correlations of $T F A M$ and $T F B 2 M$ expression profiles with the contents of flavor substances}

The association of partial flavor ingredients in LDM of JBP including IMP, IMF, Glu and oleic acid $\left(\mathrm{C}_{18: 1}\right)$ and so forth with two gene expression levels detected in the tissues were analyzed and shown in Table 7 and Table 8 . The results clearly showed that the correlation coefficients of TFAM expression level in AF, SF and LDM with $\mathrm{C}_{18: 1}$ and TUFA were all negatively significant, while the opposite occurred with SFA contents $(\mathrm{P}<0.05)$. There was also a prominently positive correlation detected between palmitic acid $\left(\mathrm{C}_{16: 0}\right)$ content, a primary kind of SFA, and the expression of TFAM mRNA in SF $(\mathrm{P}<0.05)$. Unexpectedly, distinctly negative correlation coefficients between the expression of TFAM, in liver and LDM, and IMP level were observed $(\mathrm{P}<0.05)$. Moreover, we also noticed that the levels of TFB2M and TFAM mRNA expression in LDM were all highly positively linked with the content of $\operatorname{IMF}(\mathrm{P}<0.05)$.

Table 7. The correlation analyses with the expression of TFAM gene and main flavor substance

\begin{tabular}{l|c|c|c|c|c|c|c|c}
\hline \multirow{2}{*}{ Tissues } & \multicolumn{7}{c}{ Main flavor substances } \\
\cline { 2 - 9 } & IMP & IMF & Glu & TAA & C $_{16: 0}$ & C $_{18: 1}$ & SFA & TUFA \\
\hline Liver & -0.273 & 0.200 & -0.008 & 0.036 & 0.224 & -0.219 & 0.106 & -0.106 \\
SF & 0.079 & 0.239 & -0.142 & 0.027 & $0.412^{* *}$ & $-0.349^{*}$ & $0.373^{*}$ & $-0.373^{*}$ \\
AF & 0.207 & 0.169 & -0.024 & 0.047 & 0.241 & $-0.314^{*}$ & $0.338^{*}$ & $-0.338^{*}$ \\
LDM & -0.197 & $0.582 * *$ & -0.025 & 0.166 & 0.241 & $-0.411^{* *}$ & $0.351^{*}$ & $-0.351^{*}$ \\
\hline
\end{tabular}

Superscript indicates significant difference $(* \mathrm{P}<0.05 ; * * \mathrm{P}<0.01)$.

$\mathrm{IMP}=$ inosinic acid; $\mathrm{IMF}=$ intramuscular fat; $\mathrm{Glu}=$ glutamic acid; TAA $=$ total amino acid; $\mathrm{C}_{16: 0}=$ palmitic acid; $\mathrm{C}_{18: 1}=$ oleic acid; $\mathrm{SFA}=$ saturated fatty acid; TUFA = total unsaturated fatty acid. 
Table 8 . The correlation analyses with the expression of TFB2M gene and main flavor substance

\begin{tabular}{l|c|c|c|c|c|c|c|c}
\hline \multirow{2}{*}{ Tissues } & \multicolumn{7}{c}{ Main flavor substances } \\
\cline { 2 - 9 } & IMP & IMF & Glu & TAA & C16: $^{0}$ & C $_{18 ; 1}$ & SFA & TUFA \\
\hline Liver & $-0.306^{*}$ & -0.131 & 0.174 & 0.049 & 0.009 & -0.118 & -0.071 & 0.071 \\
SF & 0.021 & 0.136 & 0.054 & -0.062 & 0.204 & -0.292 & 0.183 & -0.183 \\
AF & -0.020 & 0.077 & 0.013 & -0.097 & 0.179 & -0.291 & 0.129 & -0.129 \\
LDM & $-0.319^{*}$ & $0.523^{* *}$ & -0.101 & -0.217 & 0.252 & -0.257 & 0.246 & -0.246 \\
\hline
\end{tabular}

Superscript indicates significant difference $(* \mathrm{P}<0.05)$.

\section{Discussion}

To our knowledge, this present article represents the first cloning and characterization of full CDS regions of TFAM and TFB2M obtained from JBP. There were five mutations in nucleotide sequences, though only with two missense mutations located in amino acid sequences that were outside of $r A D c$ domain in $T F B 2 M$, suggesting that that might lead to impacts on its gene functions and further studies could be needed. HMG boxes being conserved domains of TFAM protein are widely involved in a variety of important nuclear biological functions, including the regulation of DNA replication, transcription, recombination and repairing (Reeves and Adair, 2005). Nevertheless, $r A D c$ with a property of rRNA (adenine-N6, N6-)-dimethyltransferase activity at the location of the 78th-275th amino acid residues of TFB2M enables TFB2M to be a dual-function protein which is also related to rRNA methyltransferases and possesses marked transcriptional activation properties in vitro. From the perspective of biological processes, TFB2M protein stimulates transcription in collaboration with the HMG box transcription factor, TFAM, and is homologous to rRNA methyltransferases (McCulloch and Shadel, 2003). Reflecting on the basis of this present study it was postulated that TFAM and TFB2M might play a role in a yetto-be-elucidated mechanism for regulation of adipogenesis-related gene expression thus could be associated with various phenotypic traits.

In this current study, qRT-PCR assays were used to detect the mRNA expression levels of TFAM and TFB2M in the nine tissues of JBP pigs. Results showed that the two genes were ubiquitously expressed but both were characterized by quite lower levels in lung, heart and LDM. Simultaneously, extremely significant differences were observed for two genes among these tissues. Sun (2015) finding the higher expression of TFAM gene in the pituitary gland of Guizhou White Goat hypothesized that TFAM gene might be closely related to the growth of bones and tissues due to the effect of growth hormone secreted by pituitary gland. Additionally, through the investigation of a dynamic pattern of expression of genes involved in mtDNA replication and transcription during mouse white and brown adipocyte differentiation, Murholm et al. (2009) encountered, as for TFAM and TFB2M, that their induction in white adipose tissues following cold exposure, suggests their involvement in the mitochondrial 
biogenesis occurring during the transformation to a brown adipose tissues-like depot. Not surprisingly, evidently higher two gene mRNA levels, especially for TFAM, were noted in adipose tissues compared with those in LDM, which might indicate high secretory activity of adipocyte tissues and could be linked with the impacts that TFAM and TFB2M have on the mechanism of lipogenesis. The spleen, as an immune organ, regulates humoral immunity and cellular immunity of organism. Previous work by Aharoni-Simon et al. (2011) demonstrated the consequent link of mitochondrial dysfunction with fatty liver disease in mouse. And Liu et al. (2011) also found that the number of lymphocytes in liver tissue of fatty liver decreased, implying that that had a certain impact on immune function. A possible explanation for the high transcriptional levels of the two genes obtained in spleen could also have been due to the effect of TFAM and TFB2M genes on regulation of lipid metabolism. However, there are a variety of elements modulating gene expression level including DNA methylation, histone acetylation, histone methylation, transcription factors and alternative splicing (Su et al., 2011; Robertson et al., 1999). Hence, further study is suggested to elucidate whether the mechanisms aforementioned affect the expression of TFAM and TFB2M.

Recently, increasing related studies on correlation analysis between gene expression and meat quality, such as drip loss and IMF content, have been reported in pigs (Gandolfi et al., 2011; Hamill et al., 2012). However, as for TFAM and TFB2M, two important factors of mtDNA transcription machinery, directly involved in energy production and with effects on the formation of marbling which is an indicator of cell number of intramuscular adipose tissue (Cotney et al., 2007; Holloway et al., 2009), it is the fact that studies on impacts of these genes on pork quality characteristics have been seldom reported. Considering the potential effect of TFAM and TFB2M genes, it was decided to perform an association study with meat and carcass quality traits according to their physiological roles and expression profiles in JBP pigs. Adipose, skeletal muscle and liver are the three major important tissues involved in fatty acid metabolism, thus genes expression in these tissues may be strongly related with traits like marbling score (Guo et al., 2012). Sun (2015) proposed that there were significant correlations of TFAM and TFB2M gene expression in particular tissues with meat quality and carcass traits in Guizhou goats. In the current study, analysis results inferred that there could be potential causal relationships among the expression levels of TFAM and TFB2M in these detected tissues, especially for LDM and liver tissue, and parts of carcass and meat quality traits. For two genes, significant correlations were found between transcript abundance centered in the tissues of SF, LDM and liver and several traits considered to be the most important factors that determine meat quality like marbling score, WHC, IMF and meat color (Grunert et al., 2004) (Tables 4 and 5). It was especially clear that a majority of significant correlation coefficients of the two genes obtained centered upon those fatness-related traits such as BFT, $\mathrm{FT}_{6 \sim 7}$ and $\mathrm{FP}$, which roughly corresponded to the finding obtained by Wilson-Fritch et al. (2004) concerning decreased expression level of nuclearencoded mitochondrial genes accompanying the onset of obesity in mice. Interestingly, significant negative correlations of LP and LEMA, and positive correlation of FP with the expression levels of TFAM were both noted in SF and AF tissues. These findings echoed the assumption mentioned above that the genes weigh somewhat on 
lipid metabolism and thereby impacted the yield and formation of lean muscle. In the meantime, remarkable correlations of mRNA expression of both genes in LDM with IMF content were paid attention to, which once again may indicate the certain function of the genes in metabolic activity of adipogenesis inside of muscles. In the light of the non-negligible findings that TFAM mRNA expression in adipose tissues is inextricably linked with $\mathrm{C}_{16: 0}, \mathrm{C}_{18: 1}$, SFA and TUFA it could be assumed that the biological function of gene might be involved in fatty acid synthesis and degradation, nevertheless, this will need further confirmation.

In other respects, quite a few convincing evidences that are mainly inclined to single nucleotide polymorphism (SNP) study supporting a role of TFAM and TFB2M in regulating mtDNA replication thus indirectly being likely to be responsible for fat metabolism in organism, as well as performance and production of livestock, can be found. Relevant SNPs in TFAM have been revealed to be associated, with subcutaneous fat depth and marbling scores as well as important growth, fertility, milk yield and survival traits, in beef cattle and dairy cows, respectively (Jiang et al., 2005; Clempson et al., 2011). Likewise, SNP analyses of TFB2M with goat carcass and meat quality traits have been investigated in previous study by Sun et al. (2015). In reports related to human disease, TFAM was supposed to be one of the DNA markers targeted for therapy of predisposition to obesity, while TFB2M was confirmed to be essential for mitochondrial and cellular function in pancreatic beta-cells and thus be linked with the onset of diabetes (Hudson et al., 2008; Nicholas et al., 2017). Nevertheless, further studies to verify and confirm the causative relationship between molecular mechanism of these two porcine candidate genes, TFAM and TFB2M, and part of the meat and carcass traits still remain to be explored.

\section{Conclusion}

In this research, CDS regions of the two genes were first time obtained and then analyzed by bioinformatics in JBP. In addition, qRT-PCR assay showed that there were significantly different expression levels of TFAM and TFB2M among detected tissues. And finally, correlation analyses suggested that the two genes may play certain roles in the regulation of carcass and meat quality characteristics in JBP pigs. However, further investigation on expression patterns and validation of effects of these genes on meat quality in other organisms are recommended before incorporating into the breeding program.

\section{Acknowledgements}

This work was financially supported by the Program of Breeding of New Species of Agricultural (Livestock and Poultry) in Zhejiang (2016C02054-3). The authors are grateful to Professor Qing-jun Shao and Neveen Gray for their valuable suggestions and careful proof reading of our manuscript. The authors thank Zhejiang Qinglian Food Co., Ltd for providing samples and technical assistance of their staff. 


\section{References}

A haroni-Simon M., Hann-Obercyger M., Pen S., Madar Z., Tirosh O. (2011). Fatty liver is associated with impaired activity of PPARgamma-coactivator lalpha (PGC1alpha) and mitochondrial biogenesis in mice. Lab. Invest., 91: 1018-1028.

AOAC (1990). Official Methods of Analysis of AOAC International. 5th ed. Association of Official Analytical Chemists, Washington, DC, USA.

China National Commission of Animal Genetic Resources (2011). Animal Genetic Resources in China Pigs. China Agriculture Press, Beijing.

Clempson A.M., Pollott G.E., Brickell J.S., B ourne N.E., Munce N., Wathes D.C. (2011). Polymorphisms in the autosomal genes for mitochondrial function TFAM and UCP2 are associated with performance and longevity in dairy cows. Animal, 5: 1335-1343.

Cotn e y J., Wang Z., Sha d el G.S. (2007). Relative abundance of the human mitochondrial transcription system and distinct roles for h-mtTFB1 and h-mtTFB2 in mitochondrial biogenesis and gene expression. Nucleic Acids Res., 35: 4042-4054.

C u i Z.L., Cu i Z.H., Xi a o X.D., L u o W.X., S un Y.Y. (2015). Expression of mitochondrial transcription factor B2 in different tissues of Guizhou White Goat (in Chinese). Guizhou Agr. Sci., 43: $127-129$.

D a ir a gh i D.J., Sha d el G.S., C la y t o n D.A. (1995). Human mitochondrial transcription factor A and promoter spacing integrity are required for transcription initiation. Biochim. Biophys. Acta., 1271: $127-134$.

F alkenberg M., Gas pari M., R antanen A., Trifunovic A., Lars son N.G., Gustafs s o n C.M. (2002). Mitochondrial transcription factors B1 and B2 activate transcription of human mtDNA. Nat. Genet., 31: 289-294.

F ernandez A.I.,A lves E., F ernandez A., d e P edro E., L o pez-G a r c i a M.A.,O vi lo C., R odrigue z M.C., S i 1 i o L. (2008). Mitochondrial genome polymorphisms associated with longissimus muscle composition in Iberian pigs. J. Anim. Sci., 86: 1283-1290.

F i s h e r R.P., C l a y t o n D.A. (1988). Purification and characterization of human mitochondrial transcription factor 1. Mol. Cell. Biol., 8: 3496-3509.

Gandolfi G., Cinar M.U., Ponsuksili S., Wimmers K., Tesfaye D., Looft C., Jungst H., Tholen E., Phat s a r C., S chellander K., Davoli R. (2011). Association of PPARGC1A and CAPNS1 gene polymorphisms and expression with meat quality traits in pigs. Meat Sci., 89: 478-485.

Grun ert K.G., B re d a h 1 L., B r u n s o K. (2004). Consumer perception of meat quality and implications for product development in the meat sector-a review. Meat Sci., 66: 259-272.

Gu o X., Li u X., Xu X., Wu M., Zhang X., Li Q., Li u W., Zhang Y., Wang Y., Yu Y. (2012). The expression levels of DNMT3a/3b and their relationship with meat quality in beef cattle. Mol. Biol. Rep., 39: 5473-5479.

Hamill R.M., McBryan J., McGee C., Mullen A.M., Sweeney T., Talbot A., Cairns M.T., Davey G.C. (2012). Functional analysis of muscle gene expression profiles associated with tenderness and intramuscular fat content in pork. Meat Sci., 92: 440-450.

H o 11 o w a y G.P., B o n e n A., S p r i e t L.L. (2009). Regulation of skeletal muscle mitochondrial fatty acid metabolism in lean and obese individuals. Am. J. Clin. Nutr., 89: 455S-462S.

Huds on N.J., Lehnert S.A., Harper G.S. (2008). Obese humans as economically designed feed converters: symmorphosis and low oxidative capacity skeletal muscle. Med. Hypotheses., 70: 693-697.

Jiang Z., Kunej T., Michal J.J., Gaskins C.T., Reeves J.J., Busboom J.R., Dovc P., Wrigh t R.J. (2005). Significant associations of the mitochondrial transcription factor A promoter polymorphisms with marbling and subcutaneous fat depth in Wagyu x Limousin F2 crosses. Biochem. Biophys. Res. Commun., 334: 516-523.

Jiang Z., Michal J.J., Chen J., Daniels T.F., Kunej T., Garcia M.D., Gaskins C.T., B u s b o o m J.R., A lex and e r L.J. Jr., Wright R.W., M a c N e il M.D. (2009). Discovery of novel genetic networks associated with 19 economically important traits in beef cattle. Int. J. Biol. Sci., 5: 528-542.

Kauffman R.G., Eikelenboom G., van der Wal P.G., Engel B., Zaar M. (1986). 
A comparison of methods to estimate water-holding capacity in post-rigor porcine muscle. Meat Sci., 18: 307-322.

Litonin D., S o logub M., Shi Y., S avkina M., Anikin M., F alken berg M., Gustafs s on C.M., Temiakov D. (2010). Human mitochondrial transcription revisited: only TFAM and TFB2M are required for transcription of the mitochondrial genes in vitro. J. Biol. Chem., 285: 18129-18133.

Liu Y.X., Huang Y.Z., Tong D.S., J in Y.F. (2011). Change and sense of lymphocyte subsets in peripheral blood from patients with nonalcoholic fatty liver disease (in Chinese). Mod. Med. J. China, 13: 32-34.

Livak K.J., S chmittgen T.D. (2001). Analysis of relative gene expression data using real-time quantitative PCR and the $2^{-\triangle \Delta C T}$ method. Methods, 25: 402-408.

M c Cullo ch V., Sha d e l G.S. (2003). Human mitochondrial transcription factor B1 interacts with the C-terminal activation region of h-mtTFA and stimulates transcription independently of its RNA methyltransferase activity. Mol. Cell. Biol., 23: 5816-5824.

Murholm M., Dixen K., Qvortrup K., Hansen L.H., Amri E.Z., Madsen L., Barbatelli G., Quistorff B., Hansen J.B. (2009). Dynamic regulation of genes involved in mitochondrial DNA replication and transcription during mouse brown fat cell differentiation and recruitment. Plos One, 4: e8458.

Ni cholas L.M., Valtat B., Medina A., Anders on L., A bels M., Mollet I.G., Ja in D., Elias s on L., W i e rup N., F ex M., Muld er H. (2017). Mitochondrial transcription factor B2 is essential for mitochondrial and cellular function in pancreatic beta-cells. Mol. Metab., 6: 651-663.

Rantanen A., Gaspari M., Falkenber M., Gustafs son C.M., Lars son N.G. (2003). Characterization of the mouse genes for mitochondrial transcription factors B1 and B2. Mamm. Genome., 14: 1-6.

R e b e lo A.P., D i 11 o n L.M., M or a e s C.T. (2011). Mitochondrial DNA transcription regulation and nucleoid organization. J. Inherit. Metab. Dis., 34: 941-951.

R e e ves R., A d a ir J.E. (2005). Role of high mobility group (HMG) chromatin proteins in DNA repair. DNA Repair (Amst)., 4: 926-938.

Robertson K.D., Uzvolgyi E., Liang G.N., Talmadge C., Sumegi J., Gonzale s F.A., J o n e s P.A. (1999). The human DNA methyltransferases (DNMTs) 1, 3a and 3b: coordinate mRNA expression in normal tissues and overexpression in tumors. Nucleic Acids Res., 27: 2291-2298.

S u J., Wang Y., Li u Q., Yang B., Wu Y., Lu o Y., Hu G., Zhang Y. (2011). Aberrant mRNA expression and DNA methylation levels of imprinted genes in cloned transgenic calves that died of large offspring syndrome. Livest. Sci., 141: 24-35.

S u n Y.Y. (2015). Research of expression and polymorphism of TFAM and TFB2M on meat quality traits and slaughter traits in Guizhou White Goat. Master Thesis, Guizhou University, Guizhou, China.

S un Y., L u o W., X i e H., Zh ang Y., Cai H. (2015). Analysis of association between polymorphism of TFB2M gene and meat quality, growth and slaughter traits in Guizhou White Goat, a well-known Chinese indigenous goat breed. Pak. J. Zool., 47: 1605-1610.

Wang Z., Chen Q., Li a o R., Zhang Z., Zhang X., Li u X., Zhu M., Zhang W., Xue M., Yang H., Zheng Y., Wang Q., P a n Y. (2017). Genome-wide genetic variation discovery in Chinese Taihu pig breeds using next generation sequencing. Anim. Genet., 48: 38-47.

Wilson-Fritch L., Nicoloro S., Chouinard M., Lazar M.A., Chui P.C., Leszyk J., Straubhaar J., C zech M.P., C orvera S. (2004). Mitochondrial remodeling in adipose tissue associated with obesity and treatment with rosiglitazone. J. Clin. Invest., 114: 1281-1289.

Yakubovskaya E., Meji a E., Byrnes J., Ha mbardjieva E., Garcia-Diaz M. (2010). Helix unwinding and base flipping enable human MTERF1 to terminate mitochondrial transcription. Cell, 141: 982-993.

Yang H., Ma C., Q i a o F., S ong Y., D u M. (2005). Lipolysis in intramuscular lipids during processing of traditional Xuanwei ham. Meat Sci., 71: 670-675. 